\title{
Feasibility Study Protocol: Investigating The Impact of Bleed Events On Anticoagulated Patients Diagnosed With Atrial Fibrillation Using Patient Reported Outcome Measures
}

\section{Hayley A Hutchings}

Swansea University Medical School

Kirsty Lanyon ( $\sim$ k.j.lanyon@swansea.ac.uk)

Swansea Trials Unit https://orcid.org/0000-0002-4227-6852

\section{Steven Lister}

Bristol-Myers Squibb Research

\section{Raza Alikhan}

Cardiff and Vale NHS Trust: Cardiff and Vale University Health Board

\section{Claire Fegan}

Joint Clinical Research Facility Swansea

Julian Halcox

Swansea University

\section{Gail Holland}

Swansea University College of Medicine: Swansea University Medical School

\section{Arfon Hughes}

PPI representative

\section{Rhys Jenkins}

Swansea City GP Cluster

\section{Hamish Laing}

VBHC Academy, School of Management Swansea University

Trudie Lobban

PPI Representative

\section{Lianne More}

Pfizer

\section{Diane Owen}

Swansea University College of Medicine: Swansea University Medical School

Kevin G Pollock

Bristol Myers Squibb

\section{Ceri Todd}


Kathie Wareham

Joint Clinical Researcg Facility Swansea

\section{Study Protocol}

Keywords: Atrial fibrillation, bleeding, quality of life, patient-reported outcome measures, feasibility, protocol, anticoagulation

Posted Date: July 27th, 2021

DOl: https://doi.org/10.21203/rs.3.rs-713059/v1

License: (c) (i) This work is licensed under a Creative Commons Attribution 4.0 International License. Read Full License 


\section{Abstract}

\section{Background}

Atrial fibrillation (AF) is the most common cardiac arrhythmia. Oral anticoagulation therapies (OATs) are often prescribed in conjunction with medications to restore normal heart rate rhythm which can limit the risk of an AF-related stroke and systemic thromboembolism. However, they are associated with the serious side effect of bleeding. Both clinically relevant non-major bleeding (CRNMB) and major bleeding while anticoagulated are believed to have a significant impact on patient quality of life (QoL). There is currently limited research into the effect bleeding has on QoL. The aim of this study is to evaluate the feasibility of identifying and recruiting patients diagnosed with AF, who are taking OATs and have recently experienced a bleed and collecting information on their QoL.

\section{Methods}

We will recruit a minimum of 50 patients to this cross sectional, observational study. We will recruit from general practices, secondary care and through an online AF forum. We will ask participants to complete three validated patient-reported outcome measures (PROMs: EQ5D, AFEQT and PACT-Q), approximately four weeks following a bleed and again 3 months later. We will randomly select a subset of 10 participants (of those who agree to be interviewed) to undergo a structured interview with a member of the research team to explore the impact of bleeding on their QoL and to gain feedback on the three PROMs used. We will undertake a descriptive analysis of the PROMs and demographic data. We will analyse the qualitative interviews thematically to identify key themes.

\section{Discussion}

We aim to establish if it is possible to recruit patients and use PROMs to collect information regarding how patient QoL is affected when they experience either a CRNMB or major bleed, while taking OATs for the management of AF. We will also explore the appropriateness or otherwise of the three identified PROMs for assessing quality of life following a bleed.

\section{Trial registration}

The trial has been adopted onto the NIHR Portfolio (I.D. \#47771) and registered with www.ClinicalTrials.gov (\#NCT04921176) retrospectively registered in June 2021.

\section{Background}

Atrial fibrillation (AF) is the most common cardiac arrhythmia. The overall prevalence of AF is reported to be near 3\% (Hill et al, 2019). However, many clinicians debate the true global prevalence, stating that it is likely to be underestimated as many individuals are asymptomatic and possibly undiagnosed (Nileshkumar et al, 2018). The prevalence of AF is also highest in patients aged over 60 (Darby-Stewart et al, 2012). Previous studies have concluded that for males aged 75-79, prevalence of AF doubled in 
comparison to those aged 65-69 (Chugh et al, 2014). As the general population continues to age, AF is likely to become much more of a public health burden (Xue et al, 2017), with predicted numbers of AF patients estimated to reach a minimum of 1.3 million patients in the UK by 2060 (Deirdre et al, 2017). Despite this prediction, Public Health England has already reported that between 1.3-1.5 million patients were diagnosed with AF in 2020 and it is likely that another 300-500,000 are yet to be diagnosed (Public Health England, 2021).

Patients can develop AF-related complications, for example significant risks of stroke and systemic thromboembolism and increased mortality (Hansen et al, 2018; Kannel et al, 1998). AF-related stroke as a result of untreated $A F$ is a major complication in patients and is associated with significant morbidity and mortality (Barra \& Fynn, 2015).

The general symptom burden on AF patients and the subsequent reduced ability to undertake daily activities has been widely examined in numerous studies (Montbleau et al, 2017; Thrall et al, 2006; Spertus et al, 2011). People living with AF have a much lower quality of life (QoL) than patients who do not have the disease (Barra \& Fynn, 2015).

With the steady increase in AF prevalence and its impact on patients, it is important to deliver adequate treatment and/or therapies, which can improve health related quality of life (HRQoL) in patients. Identifying any underlying causes of AF may be the first step in any treatment plan (National Clinical Guideline Centre, 2014), as treating the cause may be enough to treat and rectify the related AF. Once a detailed history of the type of AF (e.g paroxysmal or permanent) and stroke risk in the patient is established, a treatment plan should then be considered (Xu et al, 2016). Treatment plans usually involve controlling the heart rate or rhythm.

Oral anticoagulation therapies (OATs) are sometimes prescribed in conjunction with medications to restore normal heart rate rhythm in order to limit the risk of AF-related stroke and systemic thromboembolism. They are, however, associated with the serious side effect of bleeding. These bleeding complications most commonly include haematuria, intracranial, gastrointestinal, genitourinary and respiratory tract bleeding (Levine \& Goldstein, 2014; Wallis et al, 2017). Bleeding risk can be determined using calculated risk scores such as HAS-BLED (Gorman et al, 2016). A higher HAS-BLED score indicates that the patient is more at risk of a major bleed, which has previously influenced patient and clinician decisions not to offer or to withdraw anticoagulant treatment (Dar et al, 2017). However, the European Society of Cardiology 2020 guidelines advise that the benefit of anticoagulating in reducing stroke far outweighs the risk of bleeding (Hindricks et al, 2021).

Bleeding while anticoagulated is believed to have a significant impact on patient quality of life (QoL) (Res et al, 2019). Quality of life and health status are considered to have a colinear relationship, but this is typically difficult to define. There are already a multitude of validated measures which attempt to quantify Health Related Quality of Life (HRQoL) (Herdman et al, 2011; Ware et al, 1999; Badia et al, 2007). HRQoL tools can also be disease- or injury-specific and aim to capture both benefits and undesirable aspects of different diseases from a patient perspective, including consequences of living with the disease (Xiao-Jun 
et al, 2013). Extensive focus is being directed towards improving outcomes that matter to patients and their reported quality of life when delivering quality and value-based healthcare (Porter, 2013; Bae, 2015; Riva \& Pravettoni, 2016).

There is currently limited research into how bleeding events impact QoL in AF patients, especially those who experience CRNMB, for which no medical care is sought (as per Bleeding Academic Research Consortium (BARC) definition) (Mehran et al, 2011). Likewise, there are no existing and/or validated patient-reported outcome measures (PROMs) developed for use in assessing how bleeding influences patient QoL.

\section{Objectives}

- To test the feasibility of identifying patients with both CRNMB major bleeds as a result of anticoagulant treatment for AF and evaluating their QoL through both primary and secondary care settings.

- To describe and interpret the observed differences in QoL by type of bleed experienced.

- To describe the treatment received and the nature of the bleed experienced by the patient.

- To undertake structured qualitative interviews to gain detailed insight into issues from a patient perspective, for those who live with AF and have experienced a recent bleed while taking OATs.

- To evaluate the appropriateness of three chosen PROMs (EQ5D, AFEQT and PACT-Q, part 2) in capturing QoL data, specifically post a bleeding event for AF patients who are taking OATs

\section{Methods/design}

\section{Participating sites}

We will utilise three recruitment pathways for the study:

- Primary care - A large city general practice cluster made up of 8 practices in Swansea, UK

- Secondary care - A large University teaching hospital with an emergency department in Swansea, UK.

- Direct patient - An online forum and database through the Arrhythmia Alliance \& AF Association Newsletter/e-card and forums

\section{Study population}

Adult patients $(\geq 18)$ who have AF and are actively prescribed OATs and have experienced bleeds, up to a maximum of 4 weeks prior to enrolment. We will apply no upper age limit, but patients must match all other inclusion/exclusion criteria. For this feasibility study, we will select patients from the Swansea area only, who attend/have attended either anticoagulation clinics within the Swansea City Health Cluster, 
Morriston Hospital emergency department or have been admitted to wards at Morriston Hospital due to a bleed. Lastly, we will engage with people who have signed up to an online forum and support group for those diagnosed with AF, via a survey link in a circulatory newsletter and using online forums/social media.

\section{Inclusion criteria}

- Adult patients ( $\geq 18$ years old)

- Patients who have a fluent understanding of English and can therefore comprehend all study information and literature to provide fully informed consent

- Atrial fibrillation (AF) as the primary diagnosis

- Having a major or minor bleed up to a maximum of 30 days prior to point of enrolment

- Receiving oral anticoagulation therapy for AF

\section{Exclusion Criteria}

- Pregnant women

- Patients with active cancer

- Patients unable to consent for themselves

- Patients on concomitant antiplatelet therapy

\section{Patient Recruitment \& Study Sites}

Our recruitment strategies will vary considerably in order to limit unconscious recruitment bias (for example, taking care not to direct all efforts only towards those patients who are more disposed to seek medical care when experiencing bleeds). Initially, we will identify eligible patients from regular searches in secure databases in both primary (GP surgeries) and secondary care (ward/emergency department [ED]). We will send letters of invite to patients who appear eligible via primary and secondary care. No on-site visits will take place due to restrictions surrounding COVID-19 and social distancing. We will subsequently approach patients about the study by means of a face-to-face referral in clinics where viable, in line with current consultation arrangements during the COVID-19 period, or, alternatively, we will post study information to patients with AF who may not have recently visited clinics, wards, or emergency department (ED). Where appropriate, we will place study posters in locations such as general practice (GP) clinics to display information about the study. We will ensure that they are written in lay terms, to encourage discussion with GPs concerning all recent minor bleeds (as defined by ISTH), which the patient may not have thought relevant enough to mention otherwise. We will provide patient information sheets (PIS) and study cards, which will allow potential participants to take away study information for consideration, including discussion by friends and family members. We will instruct those patients interested in participating in the study to contact the clinical research team directly to streamline screening and enrolment process, and to limit time burdens on healthcare staff. 
Consent will be received digitally and an online link to the questionnaires will be sent to those who wish to participate. Follow-up data collection for these patients will also be conducted digitally.

Although the study will aim to recruit between 50 and 80 patients in the first instance, all suitable patients who are willing to partake in the study will be included. We will recruit a maximum of 200 patients.

\section{Patient Questionnaires}

We will collect basic patient demographic information on case report forms (CRFs), along with details of any bleed(s) experienced. We will ask patients additional questions relating to the ongoing management of their AF during the current Coronavirus pandemic. This will include the ease of seeking medical care in the situation of a lockdown and social distancing where visits to anticoagulation clinics are limited or entirely stopped. We will comply with Good Clinical Practice (GCP) guidelines and the General Data Protection Regulation (GDPR) in the conduct of the study and the collection of patient information.

We will ask all participants to complete three PROMs questionnaires for the study: the Euroquol 5 Dimensions-5 Levels (EQ-5D-5L) (EuroQol Group, 2009), Perception of Anticoagulant Treatment Questionnaire, Part 2 only (PACT-Q, Part 2) (Sanofi-Aventis, 2007) and Atrial fibrillation Effect on QualiTyof-life (AFEQT) (Spertus et al, 2009). We have been mindful to reduce participant burden as far as possible and have estimated that completion of all study questionnaires should take no longer than 30 minutes. We will send the participant questionnaires at two time-points for this study; an initial round will be completed at point of consent/enrolment and a second identical set of questionnaires at $90 \pm 14$ days post-enrolment. The purpose of the initial timepoint is to gather information about patient quality of life around the time of the bleed. The second time point will assess if their quality of life has changed 3 months later. We will ask participants to complete the initial round of questionnaires immediately after the consent process, to ensure that loss to follow-up is controlled. In both questionnaire sets, we will include an option for participants to take part in a further qualitative interview, after the 90-day follow up. We will send an electronic link to the initial questionnaires so the participant can access the questionnaires on a computer, tablet or phone or will provide paper copies, if requested. We will send follow-up questionnaires either through the post 3 weeks prior to the 90-day follow-up date, or we will send a survey link to participants. We will ask participants in an accompanying letter to complete and return the questionnaires within the $90 \pm 14$ day time-frame.

\section{COVID-19 Impact}

We designed the proposed study in line with current guidelines set out by the UK and Welsh Government for Coronavirus management. We will follow up to date Government guidance throughout the study (available online at https://gov.wales/coronavirus).

We acknowledge that the current COVID-19 pandemic, resulting restrictions and dramatic changes to patient lifestyle has the potential to cause additional adverse impact on QoL issues for the study population being investigated. We will not investigate QoL issues directly caused by the global Coronavirus pandemic as part of this study. However, we will collect anecdotal information about how 
patients manage their diagnosis of AF during relevant clinic closures, lockdown environment and social distancing restrictions.

\section{Data Handling \& Storage}

We will enter paper copies of CRFs and questionnaires into the study database. Questionnaires completed electronically will be saved directly into the study database. All data will be checked for consistency and accuracy.

We will host the data securely on the REDCap data management system (licensed to Swansea Trials Unit (STU)). We will hold study data anonymously within REDCap and any identifying data (including protected characteristics) will be kept separately, secured by user access restrictions.

\section{Data Analysis and Reporting}

We will focus our analysis of the questionnaire data primarily on our feasibility outcomes and present descriptive data. We will present summary information on patient demographics, mode of recruitment and levels of missing data. We will present descriptive summaries of PROMs scores reported by AF patients following a minor or major bleed while taking OATs, but we will not undertake any formal statistical comparisons. We will compare PROMs scores to age-adjusted population norms where available.

We will undertake a brief thematic analysis (Braun \& Clarke, 2006) on transcribed interview data, to draw out important common themes relating to the study question, which will not be directly captured through the chosen PROMs. We will used specific qualitative data analysis software, NVivo 12, for additional insight and to identify trends in unstructured data captured during study interviews.

\section{Ethical considerations}

This study has received ethical approval from the London Research Ethics Committee. All patients will undergo fully informed consent and have the right to withdraw at any point of the study. We recognise that that participants may become distressed or emotional during completion of study questionnaires by the recollection of previous experiences associated with bleeds. Patients may also experience low mood or anxiety associated with their AF. Our primary and secondary care researchers have clinical experience of working with patients with low mood, anxiety and distress. If a participant reports, or shows signs of low mood, distress or anxiety we will encourage them to discuss this with their primary or secondary care researcher and the participant will be signposted to local relevant services or advised to contact their GP. All data collected in the study will be stored securely within a password protected Clinical Data Management System (REDCap) while the study is conducted and anonymised for analysis purposes.

\section{Public and Patient Involvement}

We will invite two patient and public involvement (PPI) representatives to contribute to study design, selection of appropriate data collection methods/tools and to attend study steering meetings. PPI's will be selected based on appropriate relatedness to the condition, such as those currently living with $A F$ and 
prescribed anticoagulants and/or those involved with AF support groups (e.g. Arrythmia Alliance \& AF Association). We will utilise advice and expertise from PPI members of the study team to appropriately disseminate study findings.

\section{Discussion}

We will aim to determine in this feasibility study if we are able to recruit and collect QoL data from patients who have experienced either a major bleed or CRNMB, while taking OATs for the management of AF.

As this is a feasibility study, it is difficult to predict if we will successfully identify eligible patients and subsequently enrol them, based on the study inclusion/exclusion criteria. There is also uncertainty around optimal ways to identify patients promptly within the primary care and hospital settings. Patients who experience CRNMB do not always discuss these events with GPs, and identifying these patients could prove challenging, however utilising an online forum allows additional opportunity for identification and recruitment. We will test recruitment measures in this feasibility study to inform best strategies for a possible larger-scale study in the future.

\section{Abbreviations}


AFEQT Atrial Fibrillation Effect on Quality of Life

BMS Bristol Myers-Squibb

COVID- Coronavirus Disease 2019

19

CRFs Case Report Form(s)

CRNMB Clinically Relevant Non Major Bleed

ED Emergency Department

EQ5D EuroQol-5D

GCP Good Clinical Practice

GDPR General Data Protection Regulation

GP General Practitioner

HAS- $\quad$ HTN (SBP>160), Age >65, Stroke, Bleeding history or predisposition, Liver and Kidney

BLED dysfunction, Elevated or unstable INRs, Drugs and Alcohol

HRQoL Health-related Quality of Life

ISTH International Society on Thrombosis and Haemostasis

OATs Oral Anticoagulation Therapies

PACT-Q Perception of Anti-Coagulant Treatment Questionnaire

PIS Patient Information Sheet

PPI Patient and Public Involvement

PROMs Patient Reported Outcome Measure(s)

QoL Quality of Life

REDCap Research Electronic Data Capture

\section{Declarations}

\section{Ethics approval and consent to participate}

This protocol has received ethics approval from the London -Riverside Research Ethics

Committee (20/PR/0479). All participants will provide written informed consent prior to taking part in the proposed study.

\section{Consent for publication}


Participants will provide written informed consent for their anonymised data and interview quotes where relevant to be used in any future publications.

\section{Availability of data and materials}

All data collection instruments are available following an appropriate request for use license. The structured interview format is available as an additional document. Anonymised versions of the dataset used will be available from the corresponding author upon reasonable request.

\section{Competing interests}

Hayley A Hutchings leads this independent research which has received funding from Pfizer and Bristol Myers Squibb.

Hamish Laing is an employee of Swansea University who has received a grant for a Value-Based Healthcare programme of work from Pfizer Ltd., which he leads and support to attend a meeting.

Steven Lister is an employee of BMS who are funding the independent research and holds stock in BMS and Pfizer.

Kevin Pollock is an employee of BMS who have provided funding for the research.

Julian Halcox has received funding to their instruction from Pfizer and Bristol Myers Squibb since initial planning of this work, has received research grants to institution from Pfizer and Bristol Myers Squibb, personal consulting fees and payments for lectures from Pfizer and Bristol Myers Squibb, Bayer and Boehringer Ingelheim and finally support from Pfizer and Bristol Myers Squibb for attending meetings including travel costs paid to meeting organizers/travel companies.

Gail Holland, Claire Fegan, Kirsty Lanyon, Arfon Hughes, Rhys Jenkins, Trudie Lobban, Lianne More, Diane Owen, Ceri Todd and Kathie Wareham declare that they have no conflict of interest.

\section{Funding}

This study was sponsored by Pfizer and Bristol Myers Squibb, who will have oversight of all publications and conference presentations. A final report detailing the results of the study will be submitted to Pfizer and Bristol Myers Squibb upon its completion. The study is being conducted and managed through Swansea University who will act as the research governance study sponsor, independently of the Pfizer and Bristol Myers Squibb.

\section{Authors' contributions}

$\mathrm{HAH}$ is the Chief Investigator of the study. HAH and SL conceived the project and developed the initial protocol. $\mathrm{KL}$ is managing the study, developed the full protocol for ethics and governance approvals, and drafted the initial manuscript. GH provided input into protocol development and governance. All authors 
provided input into the study development and confirmed accuracy and scientific value. All authors approved the final manuscript.

\section{Acknowledgements}

Not applicable

\section{References}

1. Lancaster GA, Dodd S, Williamson PR. Design and analysis of pilot studies: recommendations for good practice. J Eval Clin Pract 2004;10(2):307-12.

2. Schulman S, Kearon C, Subcommittee on Control of Anticoagulation of the S, et al. Definition of major bleeding in clinical investigations of antihemostatic medicinal products in non-surgical patients. $J$ Thromb Haemost 2005;3(4):692-4.

3. Liu GJ, Wang YF, Chen PY, et al. The efficacy and safety of novel oral anticoagulants for the preventive treatment in Atrial fibrillation patients: a systematic review and meta-analysis. Drug Deliv 2014;21(6):43652.

4. Nileshkumar J Patel, Varunsiri Atti, Raul D Mitrani,Juan F Viles-Gonzalez, Jeffrey J Goldberger. Global rising trends of Atrial fibrillation:a major public health concern. Heart 2018;(0):1-2

5. Darby-Stewart A, Dachs R, Graber MA. Rivaroxaban vs. warfarin for stroke prevention in patients with nonvalvular Atrial fibrillation. Am Fam Physician 2012;85(6):577-86.

6. Chugh, Sumeet $S$ et al. Worldwide epidemiology of Atrial fibrillation: a Global Burden of Disease 2010 Study. Circulation2014;129(8): 837-47.

7. Xue Li, Vicki C. Tse, Lung Wai Au-Doung, lan C.K. Wong, Esther W. Chan. The impact of ischaemic stroke on Atrial fibrillation-related healthcare cost: a systematic review. EP Europace 2017;19(6):937-947

8. Deirdre A. Lane, Flemming Skjøth, Gregory Y. H. Lip, Torben B. Larsen, Dipak Kotecha. Temporal Trends in Incidence, Prevalence, and Mortality of Atrial fibrillation in Primary Care. Journal of the American Heart Association Cardiovascular and Cerebrovascular Disease 2017;6(5):e005155

9. Hansen PW, Sehested TSG, Fosbol EL, et al. Trends in warfarin use and its associations with thromboembolic and bleeding rates in a population with Atrial fibrillation between 1996 and 2011. PLoS One 2018;13(3):e0194295.

10. Kannel WB, Wolf PA, Benjamin EJ, et al. Prevalence, incidence, prognosis, and predisposing conditions for Atrial fibrillation: population-based estimates. Am J Cardiol 1998;82(8A):2N-9N. 
11. Sérgio Barra, Simon Fynn. Untreated Atrial fibrillation in the United Kingdom: Understanding the barriers and treatment options. Journal of the Saudi Heart Association 2015;27(1): 31-43

12. Kara E. Montbleau, Deandre King, Lori Henault, Jared W Magnani \& Albert Lee (Reviewing Editor). Health literacy, health-related quality of life, and Atrial fibrillation. Cogent Medicine 2017; 4(1)

13. Graham Thrall, Deirdre Lane, Douglas Carroll, Gregory Y.H. Lip. Quality of Life in Patients with Atrial fibrillation: A Systematic Review. The American Journal of Medicine 2006; 119(5) 448.e1-448.e19

14. Spertus, J., et al. Development and Validation of the Atrial fibrillation Effect on QualiTy-of-Life (AFEQT) Questionnaire in Patients With Atrial fibrillation. Circulation: Arrhythmia and Electrophysiology 2011;4(1): 15-25.

15. Hagens VE et al. Effect of rate or rhythm control on quality of life in persistent Atrial fibrillation. Results from the Rate Control Versus Electrical Cardioversion (RACE) Study. J Am Coll Cardiol. 2004;43(2):241-7.

16. National Clinical Guideline Centre. Atrial fibrillation: the management of Atrial fibrillation. Clinical guideline: Methods, evidence and recommendations. June 2014.

17. Anderson, J. L., et al. Management of Patients With Atrial fibrillation (Compilation of 2006 ACCF/AHA/ESC and 2011 ACCF/AHA/HRS Recommendations). Circulation 2013 127(18): 1916-1926.

18. January CT, Wann LS, Alpert JS, et al. 2014 AHA/ACC/HRS guideline for the management of patients with Atrial fibrillation: A report of the American College of Cardiology/American Heart Association Task Force on Practice Guidelines and the Heart Rhythm Society. J Am Coll Cardiol 2014; 64:2246-2280.

19. Xu J, Luc JG, Phan K. Atrial fibrillation: review of current treatment strategies. J Thorac Dis. 2016;8(9):E886-E900.

20. Wallis CJD, Juvet T, Lee $Y$, et al. Association Between Use of Antithrombotic Medication and Hematuria-Related Complications. JAMA 2017;318(13):1260-71.

21. Gorman EW, Perkel D, Dennis D, Yates J, Heidel RE, Wortham D. Validation Of The HAS-BLED Tool In Atrial fibrillation Patients Receiving Rivaroxaban. J Atr Fibrillation 2016;9(2):1461.

22. Dar T, Yarlagadda B, Vacek J, et al. Management of Stroke risk in Atrial fibrillation patients with bleeding on Oral Anticoagulation Therapy-Role of Left Atrial Appendage Closure, Octreotide and more. J Atr Fibrillation 2017;10(4):1729.

23. Gerhard Hindricks et al. ESC Scientific Document Group, 2020 ESC Guidelines for the diagnosis and management of Atrial fibrillation developed in collaboration with the European Association for CardioThoracic Surgery (EACTS): The Task Force for the diagnosis and management of Atrial fibrillation of the 
European Society of Cardiology (ESC) Developed with the special contribution of the European Heart Rhythm Association (EHRA) of the ESC, European Heart Journal, 2021;42(5): 373-498.

24. Res LCS, Lubberts B, Shah SH, et al. Health-related quality of life after adverse bleeding events associated with antithrombotic drug therapy - A systematic review. Hellenic J Cardiol 2019;60(1):3-10.

25. Herdman, M., Gudex, C., Lloyd, A. et al. Development and preliminary testing of the new five-level version of EQ-5D (EQ-5D-5L). Qual Life Res 2011; 20: 1727-1736.

26. Ware, J. E., Jr. (1999). SF-36 Health Survey. In M. E. Maruish (Ed.), The use of psychological testing for treatment planning and outcomes assessment:1227-1246

27. Badia, X., Arribas, F., Ormaetxe, J.M. et al. Development of a questionnaire to measure health-related quality of life (HRQoL) in patients with Atrial fibrillation (AF-QoL). Health Qual Life Outcomes 2007 5(37)

28. Xiao-Jun Lin, I-Mei Lin, Sheng-Yu Fan. Methodological issues in measuring health-related quality of life. Tzu Chi Medical Journal 2013;25(1):8-12,

29. Bae JM. Value-based medicine: concepts and application. Epidemiol Health. 2015;37:e2015014.

30. Riva S and Pravettoni G. Value-Based Model: A New Perspective in Medical Decision-making. Front. Public Health 2016 4:118

31. Mehran R, Rao SV, Bhatt DL, Gibson CM, Caixeta A, Eikelboom J, Kaul S, Wiviott SD, Menon V, Nikolsky E, Serebruany V, Valgimigli M, Vranckx P, Taggart D, Sabik JF, Cutlip DE, Krucoff MW, Ohman EM, Steg PG, White $\mathrm{H}$. Standardized bleeding definitions for cardiovascular clinical trials: a consensus report from the Bleeding Academic Research Consortium. Circulation 2011; 123: 2736-47.

32. Rodeghiero, F.; Tosetto, A.; Abshire, T.; Arnold, DM.; Coller, B.; James, P.; Neunert, C.; Lillicrap, D. ISTH/SSC bleeding assessment tool: a standardized questionnaire and a proposal for a new bleeding score for inherited bleeding disorders. J Thromb Haemost 2010;8 (9): 2063-5

33. Virginia Braun \& Victoria Clark. Using thematic analysis in psychology, Qualitative Research in Psychology, 2006 3(2): 77-101

34. Buchholz, I., Janssen, M.F., Kohlmann, T. et al. A Systematic Review of Studies Comparing the Measurement Properties of the Three-Level and Five-Level Versions of the EQ-5D. PharmacoEconomics $201836,645-661$

35. De Caterina R, Brüggenjürgen B, Darius $H$, et al. Quality of life and patient satisfaction in patients with Atrial fibrillation on stable vitamin $\mathrm{K}$ antagonist treatment or switched to a non-vitamin $\mathrm{K}$ antagonist oral anticoagulant during a 1-year follow-up: A PREFER in AF Registry substudy. Arch Cardiovasc Dis. 2018;111(2):74-84. 
36. Porter ME, Lee TH. The Strategy that will fix healthcare. Harvard Business Review October 2013

37. Public Health England. Atrial fibrillation prevalence estimates for local populations. Estimates of the number of people with Atrial fibrillation in GP practices and clinical commissioning group (CCG) areas in England. April 2020 Available at: https://www.gov.uk/government/publications/atrial-fibrillationprevalence-estimates-for-local-populations

38. Hill NR, Ayoubkhani D, McEwan P,Sugrue DM, Farooqui U, Lister S, et al. (2019) Predicting Atrial fibrillation in primary care using machine learning. PLoS ONE 14(11): e0224582

39. Michael Levine, Joshua N. Goldstein; Bleeding complications of targeted oral anticoagulants: what is the risk?. Hematology Am Soc Hematol Educ Program 2014; 2014 (1): 504-509 